Bull. Austral. Math. Soc.

11 A55, 11J 81

VOL. 38 (1988) [67-76]

\title{
THE LIMITING BEHAVIOUR OF CERTAIN SEQUENCES OF CONTINUED FRACTIONS
}

\section{David ANgell}

We investigate the set of limit points of the continued fractions

$$
\frac{1}{x_{k}+} \frac{1}{x_{k-1}+} \ldots \frac{1}{x_{1}}, \quad k=1,2,3, \ldots,
$$

where $x_{1}, x_{2}, \ldots$ is a given sequence of positive integers. We show that this set is closed, and that it may include any given countable subset of $[0,1]$ if the integers $x_{k}$ are chosen appropriately. Our main result, which has applications in transcendence theory, is that the sequence of continued fractions has no rational limit point when the sequence $\left\{\boldsymbol{x}_{k}\right\}$ of partial quotients is bounded.

\section{INTRODUCTION}

Let $\mathrm{X}=\left\{x_{k}\right\}_{k} \geqslant 1$ be a sequence of positive integers. We wish to investigate the nature of $\Lambda(X)$, the set of limit points of the sequence $\left\{Q_{k}\right\}_{k \geqslant 1}$ defined by

$$
Q_{k}=\frac{1}{x_{k}+} \frac{1}{x_{k-1}+} \ldots \frac{1}{x_{1}}
$$

Let $\xi=\frac{1}{x_{1}+} \frac{1}{x_{2}+} \cdots \in \mathbf{R}-\mathbf{Q}$. Then $Q_{k}$ is the ratio $\frac{q_{k}}{q_{k+1}}$ of the denominators of successive convergents to $\xi$, and we shall sometimes write $\Lambda(\xi)$ for $\Lambda(X)$. Conversely, any irrational number $\xi, 0<\xi<1$, uniquely determines the sequence $\mathrm{X}$ and the set $\Lambda(\mathbf{X})$.

The source of this problem lies in papers by Loxton and van der Poorten [2] and Angell [1] on functional equation methods in transcendence theory. Given a real irrational

$$
\omega=\frac{1}{a_{1}+} \frac{1}{a_{2}+} \ldots
$$

with bounded partial quotients, it was found necessary to show that the ratio $\frac{q_{k}}{q_{k+1}}$ approaches an irrational limit as $k$ tends to infinity through some suitable subsequence $\mathbf{K}$ of $\mathbf{N}$-that is, in our present notation, that $\Lambda(\omega)$ contains an irrational. In this paper, we shall first see what can be said about $\Lambda(X)$ without imposing the condition of boundedness on $X$; among our results are that $\Lambda(X)$ is a closed set, and that $X$ may be chosen in such a way that $\Lambda(X)$ contains a given countable subset of $[0,1]$. We shall then return to the bounded case. We can show what the original problem requires, and even more:

Received 17 October, 1987

Copyright Clearance Centre, linc. Serial-fee code: 0004-9729/88 \$A2.00+0.00. 
ThEOREM. If $\mathbf{X}$ is bounded then $\Lambda(\mathbf{X})$ contains no rationals.

In what follows, lower case Greek letters will denote real numbers in the interval $[0,1]$; the partial quotients in the (finite or infinite) continued fraction expansions of such numbers will be denoted by the corresponding roman letters:

$$
\alpha=\frac{1}{a_{1}+} \frac{1}{a_{2}+} \ldots
$$

The $j$-th complete quotient of $\alpha$ is written

$$
\alpha_{j}=\frac{1}{a_{j+1}+} \frac{1}{a_{j+2}+} \cdots
$$

This is valid for $j=0,1,2, \ldots$ if $\alpha$ is irrational, and for $j=0,1, \ldots, n-1$ if $\alpha=$ $\frac{1}{a_{1}+} \frac{1}{a_{2}+} \cdots \frac{1}{a_{n}}$ is rational. In the latter case we set $\alpha_{n}=0$ and we leave $\alpha_{j}$ undefined for $j>n$.

\section{EXAMPLES}

1. If $x_{k}$ is a constant $x$ for all large $k$ then $\Lambda(X)=\{\lambda\}$, where

$$
\lambda=\frac{1}{x+x+} \frac{1}{x+} \cdots=\frac{1}{2}\left(-x+\sqrt{x^{2}+4}\right)
$$

which is irrational.

2. If $\mathbf{X}$ is eventually periodic of period $p$ then

$$
\Lambda(\mathbf{X})=\left\{\left[\overline{a_{p}, a_{p-1}, \ldots, a_{1}}\right],\left[\overline{a_{p-1}, \ldots, a_{1}, a_{p}}\right],\left[\overline{a_{1}, a_{p}, \ldots, a_{2}}\right]\right\},
$$

a finite set of quadratic irrationals, and has precisely $p$ elements. Here $\left[\overline{a_{p}, \ldots, a_{1}}\right]$ denotes the periodic continued fraction

$$
\frac{1}{a_{p}+} \cdots \frac{1}{a_{1}+} \frac{1}{a_{p}+} \cdots \frac{1}{a_{1}+} \ldots
$$

3. $0 \in \Lambda(X)$ if and only if $\mathbf{X}$ is unbounded.

Proof: For any $k$,

$$
\frac{1}{x_{k}+1} \leqslant Q_{k} \leqslant \frac{1}{x_{k}}
$$

(In fact both inequalities are strict when $k \geqslant 3$.) Hence $Q_{k}$ is bounded away from zero if $\mathbf{X}$ is bounded; conversely, if some subsequence of $\mathbf{X}$ increases without limit, then the corresponding subsequence of $\left\{Q_{k}\right\}_{k \geqslant 1}$ tends to zero.

4. Similarly, $\Lambda(\mathbf{X})=\{0\}$ (that is, $\lim _{k \rightarrow \infty} x_{k} Q_{k}=0$ ) if and only if $\lim _{k \rightarrow \infty}=\infty$.

5. In fact $\lim _{k \rightarrow \infty} Q_{k}$ exists (that is, $\Lambda(X)$ is a singleton) if and only if either

(i) $\mathbf{X}$ is eventually constant; or

(ii) $\lim _{k \rightarrow \infty} x_{k}=\infty$ 
(that is, in the cases covered by examples 1 and 4 ).

Proof: We have

$$
x_{k}=\frac{1}{Q_{k}}-Q_{k-1} .
$$

If $Q_{k} \rightarrow 0$ as $k \rightarrow \infty$ then $x_{k} \rightarrow \infty$; if, on the other hand, $Q_{k}$ tends to a non-zero limit then $x_{k}$ tends to a (finite) limit. In the latter case, since each $x_{k}$ is an integer, $\mathbf{X}$ must be eventually constant. This establishes one half of the result; the converse is given by examples 1 and 4 above.

\section{SOME GenERAL RESUlts.}

It is clear by counting arguments that $\Lambda(X)$ cannot be an arbitary subset of $[0,1]$; in this section we state and prove a few properties of $\Lambda(X)$.

Definition. Two real numbers $\xi, \eta$ (not necessarily in $[0,1]$ ) are said to be equivalent if

$$
\xi=\frac{a \eta+b}{c \eta+d}
$$

for some integers, $a, b, c, d$ with $a d-b c= \pm 1$.

Lemma. Let $\mathbf{X}$ be as above and $\mathbf{Y}=\left\{y_{k}\right\}_{k \geqslant 1}$, where $y_{k}=x_{k+1}$. Then $\Lambda(\mathbf{X})=$ $\Lambda(\mathbf{Y})$

Proof: Define $Q_{k}$ as in (1) and

$$
\begin{aligned}
Q_{k}^{\prime} & =\frac{1}{y_{k}+} \frac{1}{y_{k-1}+} \cdots \frac{1}{y_{1}} \\
& =\frac{1}{x_{k+1}+} \frac{1}{x_{k}+} \cdots \frac{1}{x_{2}} .
\end{aligned}
$$

Then

$$
Q_{k+1}=\frac{x_{1} p+p^{\prime}}{x_{1} q+q^{\prime}}
$$

where $\frac{p}{q}=Q_{k}^{\prime}$, and $\frac{p_{q}^{\prime}}{q^{\top}}$ is the second last convergent in the continued fraction of $Q_{k}^{\prime}$. Therefore

$$
\begin{aligned}
\left|Q_{k+1}-Q_{k}^{\prime}\right| & =\left|Q_{k+1}-\frac{p}{q}\right|=\frac{1}{q\left(x_{1} q+q^{\prime}\right)} \quad \text { from (2) } \\
& \rightarrow 0
\end{aligned}
$$

since $q \rightarrow \infty$ as $k \rightarrow \infty$. Hence any limit point of $\left\{Q_{k}\right\}$ is a limit point of $\left\{Q_{k}^{\prime}\right\}$, and conversely. 
CoRollary. If $\xi$ and $\eta$ are equivalent irrational numbers between 0 and 1 , then $\Lambda(\xi)=\Lambda(\eta)$.

PROOF: If $\xi$ and $\eta$ are equivalent, their continued fractions have the forms

$$
\begin{aligned}
& \xi=\frac{1}{x_{1}+} \cdots \frac{1}{x_{m}+} \frac{1}{z_{1}+} \frac{1}{z_{2}+} \ldots \\
& \eta=\frac{1}{y_{1}+} \cdots \frac{1}{y_{n}+} \frac{1}{z_{1}+} \frac{1}{z_{2}+} \ldots ;
\end{aligned}
$$

by $m+n$ applications of the lemma we have

$$
\Lambda(\xi)=\Lambda(\mathbf{Z})=\Lambda(\eta)
$$

Examples 1 and 2 above follow easily from this result. The converse is false; for we can clearly construct inequivalent $\xi=\frac{1}{x_{1}+} \frac{1}{x_{2}+} \ldots$ and $\eta=\frac{1}{y_{1}+\frac{1}{y_{2}}} \ldots$ with the property $\lim _{k \rightarrow \infty} x_{k}=\lim _{k \rightarrow \infty} y_{k}=\infty$; and then we have, from example $4, \Lambda(\xi)=\Lambda(\eta)=$ $\{0\}$.

Clearly $\Lambda(\mathbf{X}) \subseteq[0,1]$; conversely, we can show by an example that any $\alpha \in[0,1]$ is in $\Lambda(X)$ for some $X$. If $\alpha=0$, see examples 3 and 4 above; otherwise, suppose first that $\alpha$ is rational and write

$$
\alpha=\frac{p}{q}=\frac{1}{a_{1}+} \cdots \frac{1}{a_{n}} .
$$

If $\eta$ is any (finite or infinite) continued fraction of the form

$$
\eta=\frac{1}{a_{1}+} \cdots \frac{1}{a_{n}+} \frac{1}{m+} \frac{1}{b_{1}+} \frac{1}{b_{2}+} \ldots
$$

then $\underset{q}{p}$ is a convergent to $\eta$ and we have

$$
\left|\frac{p}{q}-\eta\right|<\frac{1}{m q^{2}}=\frac{C}{m}
$$

since $q$ is fixed. The sequence

$$
\mathbf{X}=\left\{1, a_{n}, a_{n-1}, \ldots, a_{1}, 2, a_{n}, a_{n-1}, \ldots, a_{1}, 3, \ldots\right\}
$$

then does what is required; for if $m>0$ we have

$$
Q_{m(n+1)}=\frac{1}{a_{1}+} \cdots \frac{1}{a_{n}+} \frac{1}{m+} \cdots \frac{1}{a_{n}+1} \frac{1}{1}
$$

and by the previous result

$$
\left|\frac{p}{q}-Q_{m(n+1)}\right|<\frac{C}{m}
$$


Hence $Q_{m(n+1)} \rightarrow \alpha$ as $m \rightarrow \infty$, and $\alpha \in \Lambda(X)$. If on the other hand $\alpha=$ $\frac{1}{a_{1}+} \frac{1}{a_{2}+} \cdots \notin Q$, consider the sequence

$$
\mathrm{X}=\left\{1, a_{1}, 2, a_{2}, a_{1}, 3, a_{3}, a_{2}, a_{1}, 4, \ldots\right\}
$$

We have

$$
Q_{\frac{1}{2}\left(m^{2}+3 m\right)}=\frac{1}{a_{1}+} \cdots \frac{1}{a_{m}+m+} \frac{1}{a_{1}+1}
$$

and so

$$
\left|Q_{\frac{1}{2}\left(m^{2}+3 m\right)}-\frac{p_{m}}{q_{m}}\right|<\frac{1}{m q_{m}^{2}}
$$

where $\frac{p_{m}}{q_{m}}=\frac{1}{a_{1}+} \cdots \frac{1}{a_{m}}$. But $\left|\alpha-\frac{p_{m}}{q_{m}}\right|<\frac{1}{q_{m}^{2}}$ since $\frac{p_{m}}{q_{m}}$ is a convergent to $\alpha$; hence

$$
\left|\alpha-Q_{\frac{1}{2}\left(m^{2}+3 m^{\prime}\right)}\right|<\frac{m+1}{m q_{m}^{2}} \text {. }
$$

Thus $Q_{\frac{1}{2}\left(m^{2}+3 m\right)} \rightarrow \alpha$ as $m \rightarrow \infty$, and $\alpha \in \Lambda(X)$

It is amusing to note that in these constructions we have reversed classical procedure by using an irrational, or a "complicated" rational, as an approximation to one of its convergents.

\section{Remarks.}

1. By similar means we can construct $X$ so that $\Lambda(X)$ contains any two given numbers in $[0,1]$. For example, if $\alpha=\frac{1}{a_{1}+} \ldots \frac{1}{a_{n}}, \beta=\frac{1}{b_{1}+\frac{1}{b_{2}+}} \ldots$, choose

$$
\mathbf{X}=\left\{1, b_{1}, 1, a_{n}, \ldots, a_{1}, 2, b_{2}, b_{1}, 2, a_{n}, \ldots, a_{1}, 3, b_{3}, b_{2}, b_{1}, \ldots\right\} \text {. }
$$

Then $\alpha, \beta \in \Lambda(X)$. We can even make $\Lambda(X)$ contain a given countably infinite subset of $[0,1]$; in particular, $\Lambda(X)$ may include all rationals between 0 and 1 .

2. If $X$ is the sequence defined by (3) we can calculate $\Lambda(X)$ precisely. For $j=0,1,2, \ldots, n-1$ we have

$$
\left|Q_{m(n+1)-j}-\alpha_{j}\right|<\frac{C}{m}
$$

where $\alpha_{j}$ is as defined in Section 1 , and therefore $\alpha_{j} \in \Lambda(\mathrm{X})$. For $j=n$, moreover,

$$
\left|Q_{m(n+1)-j}\right|<\frac{1}{m}
$$

so $0 \in \Lambda(X)$. Now suppose $\lambda \in \Lambda(X)$; then some sequence $\left\{Q_{k}\right\}_{k \in K}$ converges to $\lambda$. Such a sequence contains, for some fixed $j=0,1, \ldots, n$, infinitely many terms 
$Q_{m(n+1)-j}:$ the sequence of such terms tends to the limit $\alpha_{j}$ (recall that $\alpha_{n}=0$ by definition). Hence $\lambda=\alpha_{j}$; therefore $\Lambda(X)$ is precisely the set

$$
\left\{0, \frac{1}{a_{n}}, \frac{1}{a_{n-1}+a_{n}}, \ldots, \frac{1}{a_{1}+} \ldots \frac{1}{a_{n}}\right\} .
$$

Observe that $\Lambda(\mathbf{X}) \subseteq \mathbf{Q}$

3. If $\mathbf{X}$ is the sequence (4), then the above reasoning shows that

$$
\alpha_{j}=\lim _{m \rightarrow \infty} Q_{\frac{1}{2}\left(m^{2}+3 m\right)-j}
$$

and hence $\Lambda(\mathbf{X}) \supseteq\left\{0, \alpha_{0}, \alpha_{1}, \ldots\right\}$. Furthermore, if any finite sequence $b_{1}, \ldots, b_{n}$ $(n \geqslant 1)$ occurs infinitely often in $\left\{a_{k}\right\}_{k \geqslant 1}$ then the sequence $\left\{Q_{k}\right\}_{k \geqslant 1}$ contains terms of the form

$$
\frac{1}{b_{1}+} \cdots \frac{1}{b_{n}+} \frac{1}{m+} \cdots \frac{1}{a_{1}+1} \frac{1}{1}
$$

for arbitarily large $m$; hence $\frac{1}{b_{1}+} \cdots \frac{1}{b_{n}} \in \Lambda(\mathbf{X})$. It would seem that $\Lambda(\mathbf{X})$ may contain a wide variety of numbers; we close this remark with the observation that it must contain any point of accuinulation of all the numbers mentioned so far, for we have:

Theorem. For any sequence $\mathbf{X}, \Lambda(\mathrm{X})$ is closed.

PROOF: Let $\lambda$ be an accumulation point of $\Lambda(\mathbf{X})$; write $\lambda=\lim _{i \rightarrow \infty} \xi^{(i)}, \xi^{(i)} \in$ $\Lambda(\mathrm{X})$ : without loss of generality $\xi^{(1)} \neq Q_{1}$. Define $k_{j}$ inductively by setting $k_{1}=1$ and choosing $k_{j+1}$ to be the least integer $s>k_{j}$ such that

$$
0<\left|Q_{o}-\xi^{(j+1)}\right|<\frac{1}{2}\left|Q_{k_{j}}-\xi^{(j)}\right|
$$

this is always possible since, for a given $j,\left\{Q_{k}\right\}_{k \geqslant 1}$ contains elements arbitrarily close to $\xi^{(j+1)}$. (Note that the condition $Q_{\bullet} \neq \xi^{(j+1)}$ is necessary in order that the process may continue; for the same reason we specified $Q_{k_{1}} \neq \xi^{(1)}$ above.) Then $\left\{Q_{k_{j}}\right\}_{j \geqslant 1}$ is a subsequence of $\left\{Q_{k}\right\}_{k \geqslant 1}$, and

$$
\begin{aligned}
\left|\lambda-Q_{k_{j}}\right| & \leqslant\left|\lambda-\xi^{(j)}\right|+\left(\frac{1}{2}\right)^{j-1}\left|Q_{1}-\xi^{(1)}\right| \\
& \rightarrow 0 \text { as } j \rightarrow \infty .
\end{aligned}
$$

Hence $\lambda=\lim _{j \rightarrow \infty} Q_{k_{j}} \in \Lambda(\mathbf{X})$.

COROLlarY. For some $\mathbf{X}, \Lambda(X)=[0,1]$.

Proof: As in Remark 1 above, $\Lambda(X)$ may contain every rational in $[0,1]$; but $\Lambda(\mathbf{X})$ is closed. 


\section{BoUNDED SEQUeNCES}

We have seen (example 2, p.71-72) that without the boundedness condition on $\mathrm{X}$ our original problem (to show that $\Lambda(X)$ contains an irrational number) may have no solution. However, in the bounded case the situation is different: we can prove a far stronger result than we actually require.

THEOREM. If $\mathrm{X}$ is bounded then $\Lambda(\mathrm{X})$ contains no rationals.

Proof: This is an inmediate consequence of the following theorem.

Definition. For any positive integer $M$, let $\mathbf{B}_{M}$ be the set of all real numbers in $[0,1]$ which can be expanded in a continued fraction with partial quotients at most $M$. For $0 \leqslant \xi \leqslant 1$ write

$$
\mu(\xi, M)=\inf _{\beta \in B_{M}, \beta \neq \xi}|\xi-\beta| .
$$

The following result shows that a number can well be approximated by numbers with bounded partial quotients (if and) only if it is an irrational whose partial quotients satisfy the same bound.

Theorem. Let $M$ be a positive integer. Then $\mu(\xi, M)=0$ if and only if $\xi$ is an irrational element of $\mathbf{B}_{M}$.

Proof: The converse statement is quickly proved: if $\xi \in \mathbf{B}_{m}-\mathbf{Q}$ then the convergents $\beta_{m 2}=\frac{p_{m}}{q_{m}}$ to $\xi$ satisfy

$$
\beta_{m} \in \mathbf{B}_{M}, \quad \beta_{m} \neq \xi, \quad \lim _{m \rightarrow \infty} \beta_{m}=\xi
$$

so $\mu(\xi, M)=0$. To prove the forward half of the theorem we first note that if $\xi$ and $\beta$ are (finite or infinite) continued fractions

$$
\xi=\frac{1}{x_{1}+} \frac{1}{x_{2}+} \ldots, \quad \beta=\frac{1}{b_{1}+} \frac{1}{b_{2}+} \ldots
$$

and if $x_{1}=b_{1}, \ldots, x_{m}=b_{m}$, then

$$
\begin{aligned}
|\xi-\beta| & =\left|\xi_{0}-\beta_{0}\right|=\xi_{0} \beta_{0}\left|\left(\frac{1}{\xi_{0}}-x_{1}\right)-\left(\frac{1}{\beta_{0}}-b_{1}\right)\right| \\
& =\xi_{0} \beta_{0}\left|\xi_{1}-\beta_{1}\right|=\ldots \\
& =\xi_{0} \ldots \xi_{m-1} \beta_{0} \ldots \beta_{m-1}\left|\xi_{m}-\beta_{m}\right|
\end{aligned}
$$

Supppose first that $\xi=\frac{1}{x_{1}+} \cdots \frac{1}{x_{n}} \in \mathbf{Q}$. Let

$$
\beta=\frac{1}{b_{1}+} \cdots \frac{1}{b_{n+2}+} \ldots
$$


be a finite or infinite continued fraction in $\mathbf{B}_{M}$. Since $\beta$ has at least $n+2$ partial quotients, $\beta \neq \xi$. Define $m$ to be the greatest integer $s$ such that $x_{1}=b_{1}, \ldots, x_{s}=b_{s}$. Then $m \leqslant n$ and we have

$$
\begin{aligned}
|\xi-\beta| & \geqslant \xi_{0} \ldots \xi_{m-1} \beta_{0} \ldots \beta_{m-1}\left|\xi_{m}-\beta_{m}\right| \\
& \geqslant \xi_{0} \ldots \xi_{n-1}(M+1)^{-n}\left|\xi_{m}-\beta_{m}\right|
\end{aligned}
$$

(The last step is necessary since $m$ depends on $\beta$.) We now have

(i) if $m=n$ then $\xi_{m}=0$ and

$$
\left|\xi_{n 2}-\beta_{m}\right|=\beta_{m} \geqslant \frac{1}{M+1}
$$

(ii) if $m<n$ and $x_{m+1}>b_{m+1}$ then

$$
\left|\xi_{m}-\beta_{m}\right|=\beta_{m}-\xi_{m} \geqslant \frac{1}{b_{m+1}+1+} \frac{1}{M+1}-\frac{1}{x_{m+1}}
$$

using the fact (from (5)) that $\beta$ has at least $n+2$ partial quotients. Hence

$$
\begin{aligned}
\left|\xi_{m}-\beta_{m}\right| & \geqslant \frac{(M+2)\left(x_{m+1}-b_{m+1}\right)-(M+1)}{x_{m+1}\left((M+2) b_{m+1}+(M+1)\right)} \\
& \geqslant \frac{1}{N\left(M^{2}+3 M+1\right)}
\end{aligned}
$$

where $N=\max _{1 \leqslant j \leqslant n} x_{j} ;$ and

(iii) if $m<n$ and $x_{m+1}<b_{m+1}$ then $\left|\xi_{m}-\beta_{m}\right| \geqslant \frac{1}{(N+1)\left(M^{2}+M+1\right)}$ as in (ii).

Hence

$$
\inf |\xi-\beta| \geqslant \frac{\xi_{0} \ldots \xi_{n-1}(M+1)^{-n}}{(N+1)\left(M^{2}+3 M+1\right)}=C(\xi, M)>0,
$$

where the infimum here extends over all $\beta \in \mathbf{B}_{M}$ of the form (5). Since by doing this we have excluded only finitely many elements of $\mathbf{B}_{M}$, we have

$$
\mu(\xi, M)>0
$$

Suppose, on the other hand, that $\xi$ is irrational and not in $\mathrm{B}_{M}$. Let $n$ be maximal such that $x_{1}, x_{2}, \ldots, x_{n} \leqslant M$; for any $\beta \in \mathbf{B}_{M}$ of the form

$$
\frac{1}{b_{1}+} \cdots \frac{1}{b_{n+3}+} \cdots
$$


let $m$, as before, be the greatest integer $s$ such that $x_{1}=b_{1}, \ldots, x_{s}=b_{s}$. Then $m \leqslant n$ and $(6)$ is again valid. We have

(i) if $m=n$ then

$$
\begin{aligned}
\left|\xi_{m}-\beta_{m}\right| & =\beta_{m}-\xi_{m} \geqslant \frac{1}{M+1} \frac{1}{1+} \frac{1}{M+1}-\frac{1}{M+1} \\
& =\frac{1}{(M+1)\left(M^{2}+3 M+1\right)}
\end{aligned}
$$

where we have relied on the form (7) of $\beta$; and

(ii) if $m<n$ then $\left|\xi_{m}-\beta_{m}\right| \geqslant C^{\prime}(\xi, M)$, where $C^{\prime}(\xi, M)$ is the constant of (ii) or (iii) above.

Hence

$$
\inf |\xi-\beta| \geqslant \frac{\xi_{0} \ldots \xi_{n-1}(M+1)^{-n}}{(N+1)(M+1)\left(M^{2}+3 M+1\right)}=C(\xi, M)>0,
$$

where, as for the case $\xi \in \mathbf{Q}$, the infimum excludes only finitely many values of $\beta$. This completes the proof of the theorem.

\section{Remarks.}

1. In the case where $\mathbf{X}$ is bounded, it is still possible that $\Lambda(X)$ be infinite. For example, write $\alpha^{(j)}=\frac{1}{2+} \cdots \frac{1}{2+\frac{1}{1}}, \beta^{(j)}=\frac{1}{2+} \cdots \frac{1}{2+} \frac{1}{2}$, where each continued fraction has just $j+1$ partial quotients. If

$$
\xi^{(j)}=\frac{1}{2+} \cdots \frac{1}{2+} \frac{1}{1+} \cdots
$$

is a finite or infinite continued fraction whose sequence of partial quotients begins with precisely $j$ twos, then $\xi^{(j)}$ lies in $I^{(j)}$, the closed interval between $\alpha^{(j)}$ and $\beta^{(j)}$ (that is, $I^{(j)}=\left[\alpha^{(j)}, \beta^{(j)}\right]$ or $\left[\beta^{(j)}, \alpha^{(j)}\right]$ according as $j$ is odd or even). It may be checked that these intervals are pairwise disjoint. Now let $X$ be the sequence

$$
\mathbf{X}=\{1,1,2,1,1,2,2,1,2,1,1,2,2,2,1,2,2,1,2,1, \ldots\}
$$

which contains each finite sequence $1,2, \ldots, 2$ infinitely often. Then for each $j$, infinitely many $Q_{k}$ lie in $I^{(j)}$; and hence each $I^{(j)}$ contains at least one limit point of $\left\{Q_{k}\right\}_{k \geqslant 1}$. Hence $\Lambda(X)$ is infinite. (In fact for any $j$ the sequence $\left\{Q_{k_{j n}}\right\}_{n \geqslant j}$, where $k_{j n}=1+3+6+\cdots+\frac{1}{2} n(n+1)-\frac{1}{2} j(j-1)$, lies ent.irely in $\left.I^{(j)}.\right)$

2. Apart from the limit points already mentioned in the $I^{(j)}, \Lambda(\mathrm{X})$ also contains any accumulation point of all these; one such point is easily seen to be

$$
\frac{1}{2+} \frac{1}{2+} \cdots=\sqrt{2}-1
$$


which lies in none of the $I^{(j)}$.

3. We conclude with a question: if $X$ is a bounded sequence of positive integers, can $\Lambda(X)$ be uncountable? It may help to recall that $\Lambda(X)$ is a closed set which contains no rationals.

\section{References}

[1] D.D. Angell, Mahler's Method in Transcencence Theory (Ph.D. thesis, University of New South Wales, 1987).

[2] J.H. Loxton and A.J. van der Poorten, 'Arithnetic properties of certain functions in several variables III', Bull. Austral. Math. Soc. 16 (1977), 15-47.

School of Mathematics

University of N.S.W.

Kensington, N.S.W. 2033

Australia 